\title{
Changes in nanostructure and micro-plastic properties of silicon crystals under action of magnetic fields
}

\author{
L.P. Steblenko ${ }^{1}$, A.N. Kurylyuk ${ }^{1}$, O.V. Koplak ${ }^{1}$, O.N. Krit ${ }^{1}$, V.N. Tkach ${ }^{2}$, S.N. Naumenko ${ }^{1}$ \\ ${ }^{1)}$ T. Shevchenko Kyiv National University, Physical Department, \\ 2 prosp. Akademika Glushkova, build. 1,03680Kyiv,Ukraine; e-mail: koplak_o@ukr.net \\ ${ }^{2)}$ V. Bakul' Institute for Superhard Matertials, NAS of Ukraine, 2 vul. Avtozavodskaya, 04074 Kyiv, Ukraine
}

\begin{abstract}
Experimental data obtained in this work are indicative of the fact that magnetic field stimulates processes of structural relaxation in silicon. Using the secondary-ion mass-spectrometry method, we found that the concentration of alkali metals $(\mathrm{K}, \mathrm{Na}, \mathrm{Ca})$ in the subsurface layer is essentially (4 to 5 times) increased after magnetic impact. The carbon concentration is increased, too, while the concentration of oxygen (dominant impurity in silicon) is changed in various ways. Non-homogeneous distribution of impurities results in non-uniformity of heights for nano-objects that are formed from them, which leads to non-uniformity in micro-relief and causes a respective increase of the roughness parameter. The changes in impurity composition of silicon crystals, which are caused by the magnetic influence, correlate with changes in silicon micro-plastic characteristics. In this work, we found a positive magneto-plastic effect. Most probable, the reason for braking the dislocation motion in silicon crystals after magnetic treatment is diffusion of impurities along dislocation lines, which is enhanced by magnetic field. Coagulants of diffusing.
\end{abstract}

Keywords: silicon, magnetic field, nano-cluster.

Manuscript received 05.02.10; accepted for publication 02.12.10; published online 30.12.10.

\section{Introduction}

Nowadays, requirements to quality of the structure of functional semiconductors applied in micro- and nanoelectronics are essentially increasing. In relation with it, the task both to practically use the structural defects inherent to semiconductors and to study opportunities of controlling them becomes topical.

Recently, a great interest of researchers was attracted by the idea to use weak magnetic fields (with the induction $B \leq 1 \mathrm{~T}$ ) $[1-5]$ in order to control the structure and structural-dependent properties of various weakly-magnetic materials, in particular, the semiconductor ones. A number of investigators substantiate physical principles, in accord with which spin conversion in complexes of point defects (i.e., in structural nano-clusters) lies in a base for changing the properties of weakly-magnetic crystals [6-8]. As a consequence of spin evolution, weakening and break of chemical bonds in nano-clusters take place.

Taking into account the fact that physical mechanisms related with the influence of crystal spin subsystem on its physical properties are not finally ascertained yet, it seems topical to obtain new evidences and knowledge on the role of magnetic impact in changes of the nano-structure and properties inherent to diamagnetic materials. So, the aim of this work was to study changes in the structure and micro-plastic characteristics of silicon crystals under action of magnetic fields of different kinds.

\section{Experimental}

In this work, we studied the influence of magnetic treatment (MT) on dislocationless and dislocation $\mathrm{Si}$ samples. The magnetic treatment consisted in applying weak constant magnetic field $(B=0.17 \mathrm{~T})$, alternating low-frequency $(v=50 \mathrm{~Hz}, B=0.33 \mathrm{~T})$ and highfrequency $(v=53 \mathrm{GHz})$ electromagnetic fields to the investigated $\mathrm{Si}$ crystals for some definite time.

After finishing MT, we used the methods of threecrystal X-ray diffractometry (TCXRD) and atomic force microscopy (AFM) as well as methods of electron paramagnetic resonance (EPR), secondary-ion mass- 
spectrometry (SIMS) and X-ray spectroscopy to study structural features of surface and subsurface Si layers. To investigate the features of dynamic behavior of dislocations, we used the methods of four-support bend of Si samples and selective chemical etching.

\section{Experimental results and discussion}

The experimental data obtained in this work are indicative of the processes of structural relaxation caused by magnetic field. The results depicted in Fig. 1 as illustration indicate diminishing the relief of projections at the surface of silicon samples processed by MT.
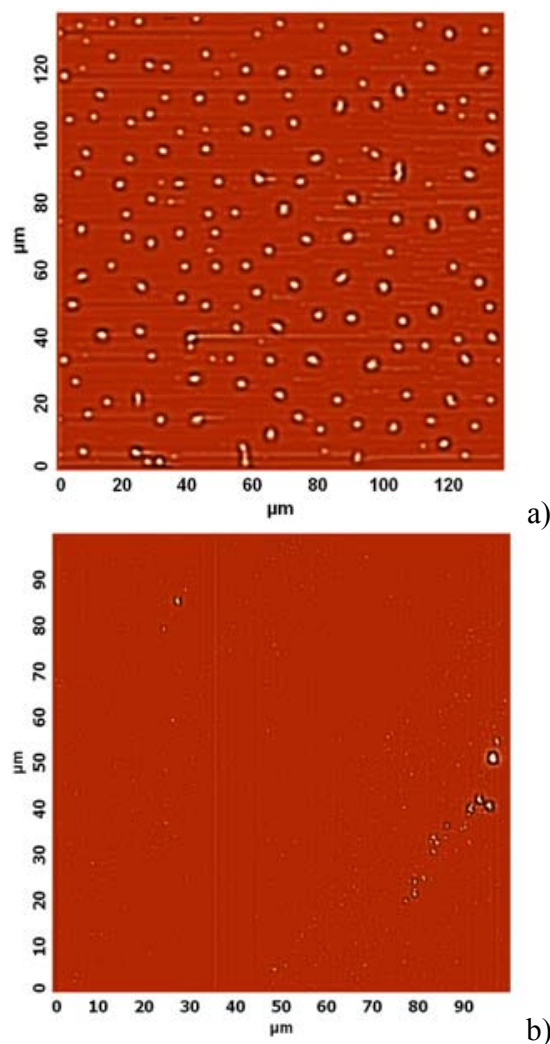

a)

Fig. 1. 2D-image of Si crystal surface structure as determined by AFM: a) initial Si samples; b) Si samples after treatment in alternating high-frequency electromagnetic field $(v=53 \mathrm{GHz}$; $t_{\mathrm{MT}}=1$ hour).

The state of surface relief after MT was estimated using the values of roughness parameter that is essentially increased (from 5 up to 40 times) after magnetic action. Reduction of the amount of relief projections on $\mathrm{Si}$ surface can be caused by spindependent processes (spin conversion) enhanced by magnetic field in nano-clusters of oxide precipitates. The change of spin configuration in the oxide precipitates that enter the composition of a natural oxide film present on Si surface is accomplished with break of the chemical bond in $\mathrm{SiO}_{\mathrm{x}}$ precipitates. As a result of nano-cluster decomposition, non-equilibrium intrinsic and impurity point defects are created.
To the number of processes initiated by magnetic field, one can relate enhancement of the adsorption process, growth in diffusion instability and intensification of inter-defect reactions. These processes force the magnetic field to stimulate not only the break of chemical bonds in structural nano-clusters but alternative redox process, too. Using the EPR method, we showed that these redox reactions stimulated by MF in silicon result in oxygen passivation of broken $\mathrm{Si}$ bonds at the boundary $\mathrm{Si}-\mathrm{SiO}_{2}$ (the so-called $\mathrm{p}_{\mathrm{B}}$-centers that are paramagnetic), and, consequently, the concentration of $p_{B}$-centers is decreased. As shown by results obtained using the TCXRD method, passivation of broken bonds on the magnetically-activated Si surface results in reduction of Debye-Waller factor. The latter is indicative of ordering the Si surface. On the other hand, experimental facts show that in parallel with this ordering some other effects take place under magnetic action. It turns out that enhancement of surface chemical activity caused by MT is accompanied by formation of high oxide nano-structural objects. As a consequence, the two-fold increase in the oxide coating thickness, changes in surface topology and, respectively, in the value of roughness parameter take place.

It is revealed in this work that magnetic field not only modifies topology and the structural state of the surface but essentially changes the impurity state of the subsurface layer, too. Our analysis with application of the SIMS method showed that the concentration of alkali metals in the subsurface layer is significantly (by 4 to 5 times) increased after MT. Using the X-ray spectroscopy method, we found that after MT the carbon concentration is sharply increased, and the concentrations of oxygen - dominating impurity in silicon - as well as silicon (basic element of this matrix) are changed, too (see Table 1). In this case, the above changes are characterized by a large dispersion of impurity concentrations fixed in various parts of the surface of Si crystals treated with magnetic field.

Table 1. Concentrations of carbon, oxygen and silicon in the studied samples before the beginning and after finishing the magnetic treatment in alternating electromagnetic high-frequency field.

\begin{tabular}{|c|c|c|c|c|}
\hline $\begin{array}{l}\text { Sample } \\
\text { number }\end{array}$ & Sample & \multicolumn{3}{|c|}{ Concentration, wt \% } \\
\hline & & $\mathrm{C}$ & $\mathrm{O}$ & $\mathrm{Si}$ \\
\hline 1 & $\begin{array}{c}\text { Silicon (reference } \\
\text { sample) }\end{array}$ & 2.66 & 0.33 & 97.00 \\
\hline $2 *$ & \multirow{5}{*}{$\begin{array}{c}\text { Silicon, after } \\
\text { magnetic treatment } \\
(v=53 \mathrm{GHz}, \\
\left.\mathrm{t}_{\mathrm{MT}}=3 \text { hours }\right)\end{array}$} & 25.21 & 0.15 & 74.64 \\
\hline $3 *$ & & 19.19 & 0.97 & 79.84 \\
\hline $4^{*}$ & & 19.48 & 0,52 & 80.00 \\
\hline $5^{*}$ & & 18.13 & 0.00 & 81.87 \\
\hline $6^{*}$ & & 7.06 & 0.99 & 91.94 \\
\hline
\end{tabular}

*Note: 2 to 6 are related to different parts of the Si sample surface after magnetic treatment. 
It cannot be excluded that non-uniform character of impurity and matrix element distributions causes nonuniformities in heights of nano-objects that are formed from them. In their turn, non-uniformities in the nanoobject height cause those in micro-relief and results in the respective growth of the roughness parameter. The found features of the structure and changes in the impurity state of Si crystals allowed to assume the possibility of changes in Si micro-plastic characteristics under magnetic field action. This assumption was confirmed by the data of experimental investigations concerning the dynamical behavior of dislocations. It was found that in silicon crystals undergone to preliminary MT, independently of magnetic field characteristics, one can observe the positive magneto-plastic effect (MPE) in the course of further mechanical deforming. The typical features of this effect are growth of the delay time for dislocation motion and increase in start tensions necessary for this motion (Figs $2 \mathrm{a}$ and $2 \mathrm{~b}$ ). As it follows from comparison of Figs $2 \mathrm{a}$ and $2 \mathrm{~b}$, the delay times for the beginning of dislocation motion vanish when the deforming tension increases from 63.5 up to $93.5 \mathrm{MPa}$. However, in Si crystals after MT, even for the increased start tensions, the dislocation free path is 3 times less than that in reference $\mathrm{Si}$ samples without MT.
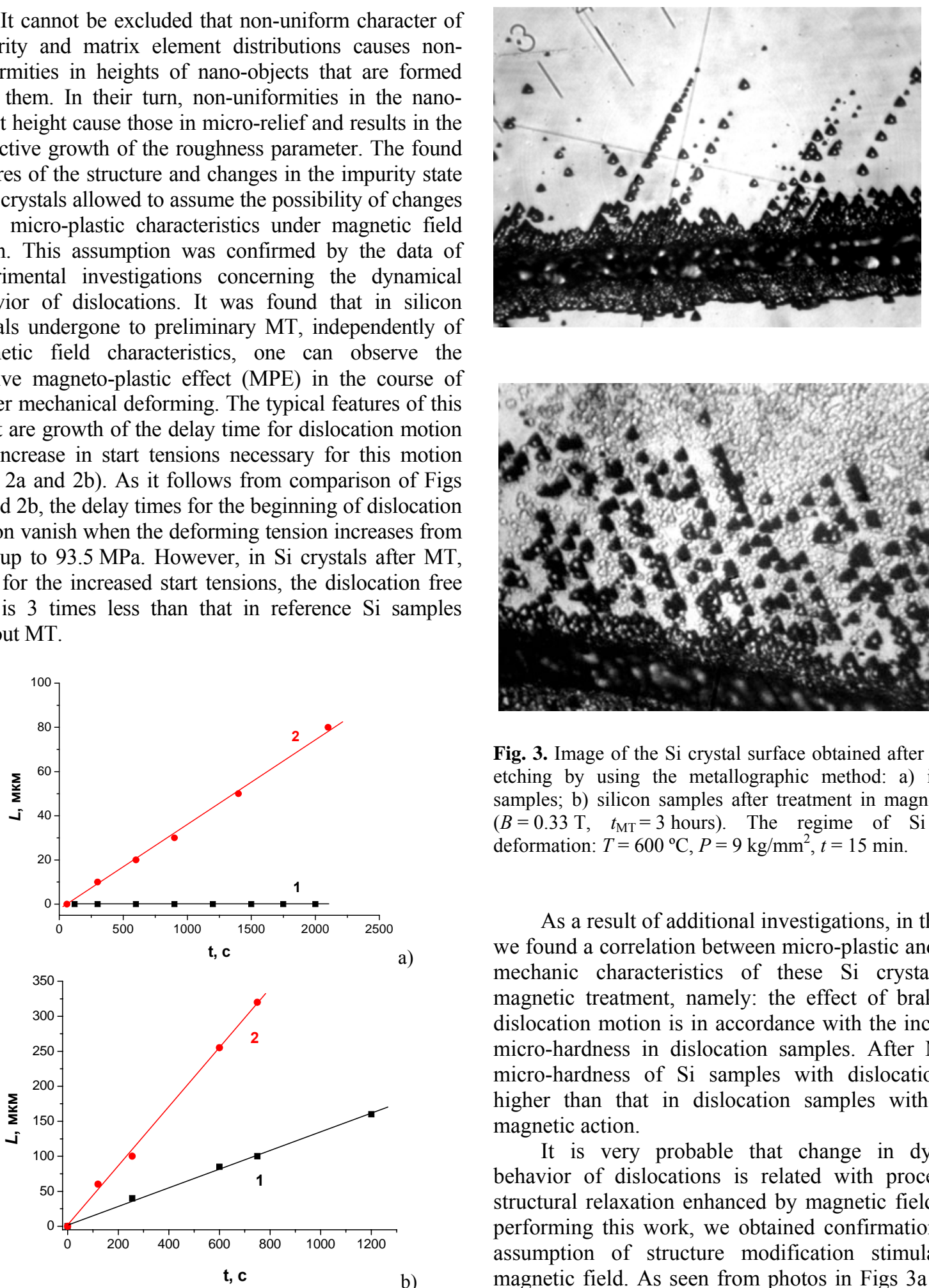

a)

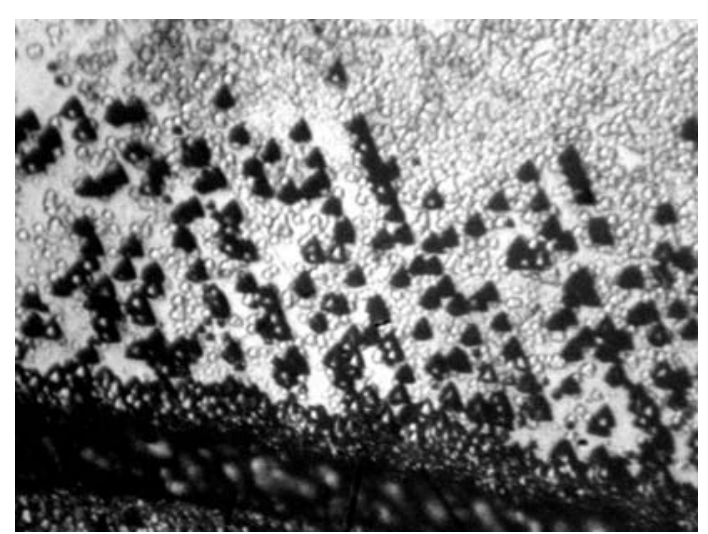

b)

Fig. 3. Image of the Si crystal surface obtained after chemical etching by using the metallographic method: a) initial $\mathrm{Si}$ samples; b) silicon samples after treatment in magnetic field ( $B=0.33 \mathrm{~T}, t_{\mathrm{MT}}=3$ hours). The regime of $\mathrm{Si}$ sample deformation: $T=600^{\circ} \mathrm{C}, P=9 \mathrm{~kg} / \mathrm{mm}^{2}, t=15 \mathrm{~min}$.

As a result of additional investigations, in this work we found a correlation between micro-plastic and micromechanic characteristics of these $\mathrm{Si}$ crystals after magnetic treatment, namely: the effect of braking the dislocation motion is in accordance with the increase of micro-hardness in dislocation samples. After MT, the micro-hardness of $\mathrm{Si}$ samples with dislocations was higher than that in dislocation samples without any magnetic action.

It is very probable that change in dynamical behavior of dislocations is related with processes of structural relaxation enhanced by magnetic field. When performing this work, we obtained confirmation of the assumption of structure modification stimulated by magnetic field. As seen from photos in Figs 3a and 3b, the state of the Si surface after MT essentially differs from that of the samples without any MT.

Contrary to the initial Si samples, those after MT are characterized with presence of flat shallow etching pits on the surface. As a rule, these pits are identified with presence of coagulants consisting of diffusing impurities in subsurface layers of these crystals. As a consequence, one can draw the following conclusion. 
The reason for the found effect of braking the dislocation motion in $\mathrm{Si}$ crystals after MT, a most probable, is enhanced by magnetic field diffusion of impurities along dislocation lines. Coagulants of diffusing impurities force formation of impurity atmospheres that slow down the dislocation motion.

\section{References}

1. V.N. Buzykin, O.I. Datsko, S.N. Postnikov. Processes of long-term relaxation in a real silicon structure after treatment with pulsed magnetic field // Elektronnaya obrabotka materialov. Kishin'ov: Academy of Sciences of Moldova Republic (Institute for Applied Physics), 2, p.16-19 (1993), in Russian.

2. A.G. Kadmensky, S.G. Kadmensky, M.N. Levin et al. Relaxation processes in MDS elements of integrated circuits caused by ionized radiation and pulsed magnetic field // Pis'ma v ZhTF, 19(3), p. 41 - 45 (1993), in Russian.
3. M.N. Levin, B.A. Zon, Magnetic field induced generation of A-like centers in Cz-Si crystals // Phys. Lett. A. 260, p. 386-390, (1999).

4. Yu.I. Golovin. Magnetoplasticity of solids // Fizika tverdogo tela, 46(5), p. 769-803, (2004), in Russian.

5. Yu.I. Golovin, R.B. Morgunov, A. A.Dmitrievskii, Influence of weak magnetic field on spindependent relaxation of structural defects in diamagnetic crystals // Materials Science and Engineering A, 15(2), Vol. 288, p. 261-265 (2000).

6. K.M. Salikhov, Yu. N. Molin, R.Z. Sagdeev and A.L. Buchachenko, Spin polarization and magnetic field effects in radical reactions, edited by $\mathrm{Yu}$. $\mathrm{N}$. Molin ElseVier, Amsterdam (1984).

7. M.I. Molotsky. Negative magnetoplastic effect in non-magnetic crystals // Fizika tverdogo tela, 35(11), p. 11-14 (1993), in Russian.

8. A.L. Buchachenko. Physical kinetics of magnetoplasticity in diamagnetic crystals // Zhurnal eksperimental'noi $i$ teoreticheskoi fiziki, 132(4), p. 827-830 (2007), in Russian. 